\title{
Author Correction: 547 transcriptomes from 44 brain areas reveal features of the aging brain in non-human primates
}

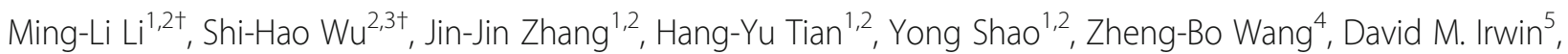
Jia-Li Li $i^{3}$ Xin-Tian $\mathrm{Hu}^{3,6,7,8^{*}}$ and Dong-Dong $\mathrm{Wu}^{1,7,9^{*}}$

\section{Correction to: Genome Biol (2019) 20:258 \\ https://doi.org/10.1186/s13059-019-1866-1}

Following publication of the original paper [1], the authors reported an error in the affiliation of Xin-Tian $\mathrm{Hu}$, who is also affiliated with "Kunming Primate Research Center, Kunming Institute of Zoology, Chinese Academy of Sciences, Kunming, Yunnan, China".

\begin{abstract}
Author details
'State Key Laboratory of Genetic Resources and Evolution, Kunming Institute of Zoology, Chinese Academy of Sciences, Kunming 650223, Yunnan, China. ${ }^{2}$ Kunming College of Life Science, University of the Chinese Academy of Sciences, Kunming 650223, Yunnan, China. ${ }^{3}$ Key Laboratory of Animal Models and Human Disease Mechanisms of Chinese Academy of Sciences \& Yunnan Province, Kunming Institute of Zoology, Chinese Academy of Sciences, Kunming 650223, Yunnan, China. ${ }^{4}$ Yunnan Key Laboratory of Primate Biomedicine Research, Institute of Primate Translational Medicine, Kunming University of Science and Technology, Kunming 650500, Yunnan, China. ${ }^{5}$ Department of Laboratory Medicine and Pathobiology, University of Toronto, Toronto, ON, Canada. ${ }^{6}$ Center for Excellence in Brain Science and Intelligence Technology, Chinese Academy of Sciences, Shanghai 200031, China. ${ }^{7}$ National Research Facility for Phenotypic and Genetic Analysis of Model Animals, Kunming Institute of Zoology, Chinese Academy of Sciences, Kunming 650223, Yunnan, China. ${ }^{8}$ Kunming Primate Research Center, Kunming Institute of Zoology, Chinese Academy of Sciences, Kunming, Yunnan, China. ${ }^{9}$ Center for Excellence in Animal Evolution and Genetics, Chinese Academy of Sciences, Kunming 650223, Yunnan, China.
\end{abstract}

Published online: 24 February 2020

\section{Reference}

1. Li M, Wu S, Zhang J, et al. 547 transcriptomes from 44 brain areas reveal features of the aging brain in non-human primates. Genome Biol. 2019;20: $258 \mathrm{https}: / /$ doi.org/10.1186/s13059-019-1866-1.

The original article can be found online at https://doi.org/10.1186/s13059019-1866-1

*Correspondence: xthu@mail.kiz.ac.cn; wudongdong@mail.kiz.ac.cn

${ }^{\dagger}$ Ming-Li Li and Shi-Hao Wu contributed equally to this work.

${ }^{3}$ Key Laboratory of Animal Models and Human Disease Mechanisms of Chinese Academy of Sciences \& Yunnan Province, Kunming Institute of Zoology, Chinese Academy of Sciences, Kunming 650223, Yunnan, China 'State Key Laboratory of Genetic Resources and Evolution, Kunming Institute of Zoology, Chinese Academy of Sciences, Kunming 650223, Yunnan, China Full list of author information is available at the end of the article

(c) The Author(s). 2020 Open Access This article is distributed under the terms of the Creative Commons Attribution 4.0 International License (http://creativecommons.org/licenses/by/4.0/), which permits unrestricted use, distribution, and reproduction in any medium, provided you give appropriate credit to the original author(s) and the source, provide a link to the Creative Commons license, and indicate if changes were made. The Creative Commons Public Domain Dedication waiver (http://creativecommons.org/publicdomain/zero/1.0/) applies to the data made available in this article, unless otherwise stated. 